Homology, Homotopy and Applications, vol.21(2), 2019, pp.107-130

\title{
TOPOLOGICAL COMPLEXITY OF A MAP
}

\author{
PETAR PAVEŠIĆ \\ (communicated by Donald M. Davis)
}

\begin{abstract}
We study certain topological problems that are inspired by applications to autonomous robot manipulation. Consider a continuous map $f: X \rightarrow Y$, where $f$ can be a kinematic map from the configuration space $X$ to the working space $Y$ of a robot arm or a similar mechanism. Then one can associate to $f$ a number $\mathrm{TC}(f)$, which is, roughly speaking, the minimal number of continuous rules that are necessary to construct a complete manipulation algorithm for the device. Examples show that $\mathrm{TC}(f)$ is very sensitive to small perturbations of $f$ and that its value depends heavily on the singularities of $f$. This fact considerably complicates the computations, so we focus here on estimates of $\mathrm{TC}(f)$ that can be expressed in terms of homotopy invariants of spaces $X$ and $Y$, or that are valid if $f$ satisfies some additional assumptions like, for example, being a fibration.

Some of the main results are the derivation of a general upper bound for $\mathrm{TC}(f)$, invariance of $\mathrm{TC}(f)$ with respect to deformations of the domain and codomain, proof that $\mathrm{TC}(f)$ is a $\mathrm{FHE}-$ invariant, and the description of a cohomological lower bound for $\mathrm{TC}(f)$. Furthermore, if $f$ is a fibration we derive more precise estimates for $\mathrm{TC}(f)$ in terms of the Lusternik-Schnirelmann category and the topological complexity of $X$ and $Y$. We also obtain some results for the important special case of covering projections.
\end{abstract}

\section{Introduction}

In 2003 Michael Farber [4] introduced the topological complexity of a space $X$, denoted $\mathrm{TC}(X)$, as a homotopy-invariant measure of the difficulty to plan a continuous motion of a robot in the space $X$. Over the years the interest for applications of topological complexity and related concepts to problems in robotics grew into an independent field of research. Topological complexity of a map is a natural extension of $\mathrm{TC}(X)$ suggested by Alexander Dranishnikov during the conference on Applied Algebraic Topology in Castro Urdiales (Spain, 2014). The new concept opens the

The author was supported by the Slovenian Research Agency research grant P1-0292 and research project J1-7025.

Received August 31, 2018, revised October 26, 2018; published on February 13, 2019.

2010 Mathematics Subject Classification: Primary 55M99; Secondary 70B15, 68T40.

Key words and phrases: topological complexity, robotics, kinematic map, fibration, covering.

Article available at http://dx.doi.org/10.4310/HHA.2019.v21.n2.a7

Copyright (C) 2019, International Press. Permission to copy for private use granted. 
possibility to build some new interesting models in topological robotics. For example, the present author used the topological complexity of a map in in [10] as a measure of manipulation complexity of a robotic device. That point of view was further developed in [11]. The main thrust of both papers was on applications to kinematic maps that arise in commonly used robot configurations. As a consequence, many related theoretical question were left aside. The purpose of the present paper is to fill that gap.

Let $f: X \rightarrow Y$ be a continuous map: given $x \in X, y \in Y$, we look for a path $\alpha=\alpha(x, y)$ in $X$ starting at $x$ and ending at a point that is mapped to $y$ by $f$. We normally assume that $X$ is path-connected and that $f$ is surjective, so that the above problem always has a solution. However, we want the assignment $(x, y) \mapsto \alpha(x, y)$ to satisfy an additional condition, namely to be as continuous as possible. More formally, we consider the space $X^{I}$ of all paths in $X$ and the projection map

$$
\pi_{f}: X^{I} \rightarrow X \times Y, \text { where } \pi_{f}(\alpha)=(\alpha(0), f(\alpha(1))) .
$$

Then every solution to the above-mentioned problem can be interpreted as a section $s: X \times Y \rightarrow X^{I}$ to the projection $\pi_{f}$. There are simple examples of maps $f: X \rightarrow Y$ such that $\pi_{f}$ does not admit a section that is continuous on entire $X \times Y$. Therefore, one may attempt to split $X \times Y$ into subspaces, each admitting a continuous section to $\pi_{f}$. The minimal number of elements in such a partition is the topological complexity of the map $f$.

Topological complexity of a map can be viewed as a natural generalization of the topological complexity of a single space, introduced by Farber [4]. However, computation of $\mathrm{TC}(f)$ requires the study of a host of new phenomena related to its domain, codomain and singularities.

In this paper we will not be concerned with the applications of $\mathrm{TC}(f)$ to robotics. Nevertheless to give a flavour of the maps which one may want to study, we just mention a variety of situations that can be modelled by $\mathrm{TC}(f)$ (see [11, Section 5] for more details).

- If $X$ is the configuration space of a system and $f: X \rightarrow Y$ is a projection to the configuration space of a part or a subsystem, then $\mathrm{TC}(f)$ measures the complexity of manipulation of the components of a complex mechanism (e.g a moving platform), where one is only interested in the positioning of some intermediate part of the structure (e.g. an object on the platform);

- The complexity of manipulation of a robotic arm is modelled by letting $X$ be a joint space, $Y$ the working space and $f: X \rightarrow Y$ the forward kinematic map of the arm (see [10] for a detailed discussion);

- Let $X$ be a configuration space of a robotic mechanism where different points of $X$ (i.e. positions of the mechanism) are functionally equivalent (e.g. for grasping, pointing, ...). If we express functional equivalence in terms of the action of some symmetry group $G$, then the manipulation complexity of the device is modelled by the topological complexity of the quotient map $X \rightarrow X / G$.

We begin the paper with a discussion of the 'correct' definition of the complexity of a map. In fact, a straightforward generalization of the standard definition of topological complexity of a space proposed by Dranishnikov turned out to be somewhat inadequate for maps with singularities. We devised an alternative approach which is 
equivalent to Dranishnikov's when applied to fibrations but yields more satisfactory results for general maps.

The third section is dedicated to a various upper and lower estimates for the topological complexity of a map. Some of these are valid for arbitrary maps, while other hold for maps that have some additional properties, e.g. are fibrations or admit a section (see Section 3.6 for a summary of main results).

In the final section we specialize to maps that are fibrations and express their complexity in terms of other homotopy invariants. This allows computation of topological complexity of many standard fibrations. In particular we show that topological complexities of covering projections can be viewed as approximations of topological complexity of the base space.

\section{Definition of $\mathrm{TC}(f)$}

We are going to define the topological complexity of a map in a way that will allow a comparison with two other related concepts - cat $(X)$, the Lusternik-Schnirelmann category of $X$, and $\mathrm{TC}(X)$, the topological complexity of $X$. In fact all three concepts can be expressed in terms of sectional numbers of certain maps.

Let $p: E \rightarrow B$ be a continuous surjection. A section of $p$ is a right inverse of $p$ i.e., a map $s: B \rightarrow E$, such that $p \circ s=1_{B}$. Moreover, given a subspace $A \subset B$, a partial section of $p$ over $A$ is a section of the restriction map $p: p^{-1}(A) \rightarrow A$. If $p$ does not admit a continuous section, it may still happen that it admits sufficiently many continuous partial sections so that their domains cover $B$.

We define $\sec (p)$, the sectional number of $p$ to be the minimal integer $n$ for which there exists an increasing sequence of open subsets

$$
\emptyset=U_{0} \subset U_{1} \subset U_{2} \subset \cdots \subset U_{k}=B,
$$

such that each difference $U_{i}-U_{i-1}, i=1, \ldots, n$ admits a continuous partial section to $p$. If there is no such integer $n$, then we let $\sec (p)=\infty$.

A word of warning is in order here, since the above is not the entirely standard definition of sectional number. Indeed, sectional number is more commonly defined as the minimal number of elements in an open cover of $B$, such that each element admits a continuous partial section to $p$. Let us denote this $\operatorname{second}$ quantity as $\sec _{\mathrm{op}}(p)$. Obviously $\sec (p) \leqslant \sec _{\mathrm{op}}(p)$. On the other hand, it is easy to see that if $p$ is a fibration and $B$ is an ANR space, then $\sec (p)$ and $\sec _{\mathrm{op}}(p)$ actually coincide. One should also note the similarity between $\sec _{\mathrm{op}}(p)$ and secat $(p)$, the sectional category of $X$ (also called Schwarz genus of $p$, cf. [13], [1]). The latter counts the minimal number of homotopy sections of $p$, therefore $\sec _{\mathrm{op}}(p)=\operatorname{secat}(p)$ if $p$ is a fibration, but in general $\sec (p)$ can be much bigger than $\operatorname{secat}(p)$ (see [11, Section 5] for some specific examples).

We are now ready to state the definition of the Lusternik-Schnirelmann category and the definitions of the topological complexity of a space and of a map. For any space $X$ let $X^{I}$ be the space of all continuous paths in $X$ (endowed with the compactopen topology) and let $P X$ be the subspace of all based paths in $X$ starting at some fixed base-point $x_{0} \in X$ (which we omit from the notation). It is well known that for 
any point $c \in[0,1]$ the evaluation map

$$
\mathrm{ev}_{c}: X^{I} \rightarrow X, \quad \alpha \mapsto \alpha(c)
$$

is a fibration (and similarly for $P X$ in place of $X$, provided that $c \in(0,1]$ ).

The Lusternik-Schnirelmann category of a space $X$ is defined as

$$
\operatorname{cat}(X)=\sec \left(\mathrm{ev}_{1}: P X \rightarrow X\right) .
$$

If $X$ is an ANR, then our definition is equivalent to the standard one that uses open coverings of $X$ by categorical subsets. For the convenience of the reader we list in the next proposition the most important properties of the Lusternik-Scnirelmann category

\section{Proposition 2.1.}

1. $\operatorname{cat}(X)=1$ if, and only if $X$ is contractible;

2. Homotopy invariance: $X \simeq Y \Rightarrow \operatorname{cat}(X)=\operatorname{cat}(Y)$;

3. Dimension-connectivity estimate: if $X$ is d-dimensional and $(c-1)$-connected, then $\operatorname{cat}(X) \leqslant \frac{d}{c}+1$;

4. Cohomological estimate: $\operatorname{cat}(X) \geqslant \operatorname{nil} \widetilde{H}^{*}(X)$, where $\widetilde{H}^{*}(X)$ is the ideal of positive-dimensional cohomology classes in $H^{*}(X)$;

5. Product formula: $\operatorname{cat}(X \times Y) \leqslant \operatorname{cat}(X)+\operatorname{cat}(Y)-1$.

More recently M. Farber [4] introduced the concept of a topological complexity of a space in order to provide a crude measure of the complexity of motion planning of mechanical systems, e.g. robot arms. The topological complexity of a (path-connected) space $X$ is

$$
\mathrm{TC}(X):=\sec (\pi), \quad \text { where } \pi=\left(e v_{0}, \mathrm{ev}_{1}\right): X^{I} \rightarrow X \times X .
$$

As before, if $X$ is an ANR space, then the above coincides with the Farber's original definition (cf. [6] or $[\mathbf{9}]$ ). It is not surprising that many properties of $\mathrm{TC}(X)$ resemble those of $\operatorname{cat}(X)$ and that the two quantities are closely related. The main properties of $\mathrm{TC}(X)$ are listed in the following proposition.

\section{Proposition 2.2.}

1. $\mathrm{TC}(X)=1$ if, and only if $X$ is contractible;

2. Homotopy invariance: $X \simeq Y \Rightarrow \mathrm{TC}(X)=\mathrm{TC}(Y)$;

3. Category estimate: $\operatorname{cat}(X) \leqslant \mathrm{TC}(X) \leqslant \operatorname{cat}(X \times X)$;

4. If $X$ is a topological group, then $\mathrm{TC}(X)=\operatorname{cat}(X)$;

5. Cohomological estimate: $\operatorname{cat}(X) \geqslant \operatorname{nil}\left(\operatorname{Ker} \Delta^{*}\right)$, where $\Delta^{*}: \widetilde{H}^{*}(X \times X) \rightarrow H^{*}(X)$ is induced by the diagonal $\Delta: X \rightarrow X \times X$;

6. Product formula: $\mathrm{TC}(X \times Y) \leqslant \mathrm{TC}(X)+\mathrm{TC}(Y)-1$.

We may finally turn to the definition of the topological complexity of a map. Let $f: X \rightarrow Y$ be a continuous surjection between path-connected spaces, and let

$$
\pi_{f}: X^{I} \rightarrow X \times Y
$$

be defined as $\pi_{f}:=\left(\mathrm{ev}_{0}, f \circ \mathrm{ev}_{1}\right)=(1 \times f) \circ \pi$. Then the topological complexity of 
the map $f$ is defined as

$$
\mathrm{TC}(f):=\sec \left(\pi_{f}\right) .
$$

Clearly $\operatorname{TC}\left(\operatorname{id}_{X}\right)=\mathrm{TC}(X)$, so the topological complexity of a map is a generalization of the topological complexity of a single space. We will see later (Example 4.10) that $\operatorname{cat}(X)=\mathrm{TC}\left(\mathrm{ev}_{1}: P X \rightarrow X\right)$, so the topological complexity of a map generalizes the Lusternik-Schnirelmann category as well.

Most of Section 3 is dedicated to the appropriate extensions of Propositions 2.1 and 2.2 for the topological complexity of a map. In the rest of this section we will relate $\mathrm{TC}(f)$ to (partial) sections of $f$, and explain why a definition of $\mathrm{TC}(f)$ based on partial sections over open covers of $X \times Y$ does not work well in general.

Let $A \subset X \times Y$, such that $A$ admits a partial section of $\pi_{f}$, say $s: A \rightarrow X^{I}$. For a fixed $x_{0} \in X$, let $\hat{A}=\left\{y \in Y \mid\left(x_{0}, y\right) \in A\right\}$ and define $\hat{s}: \hat{A} \rightarrow X$ by $\hat{s}(y)=s\left(x_{0}, y\right)(1)$. Clearly, $\hat{s}$ is a continuous partial section of $f$. Some of the consequences of this follow:

- If $\pi_{f}: X^{I} \rightarrow X \times Y$ admits a global continuous section, then so does $f: X \rightarrow Y$, i.e. $f$ is essentially a retraction of $X$ to $Y$. This immediately gives plenty of maps whose complexity is bigger than 1 . For example, the map $f:[0,3] \rightarrow[0,2]$ given by

$$
f(t):= \begin{cases}t & t \in[0,1] \\ 1 & t \in[1,2] \\ t-1 & t \in[2,3]\end{cases}
$$

(see Figure 1) clearly does not admit a section, therefore its topological complexity must be bigger than one. Compare [11, Section 5] for a general procedure for constructing maps with contractible domain and codomain and with arbitrarily high topological complexity.

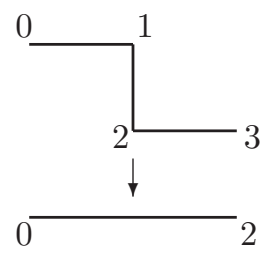

Figure 1: Map whose complexity is bigger than one.

- If $\left(x_{0}, y_{0}\right)$ is an interior point of $A$, then the above formula yields a partial section for $f$ defined on a neighborhood of $y_{0}$. This raises the question of admissible domains for partial sections of $\pi$. In particular, if $f$ is not locally sectionable at some point, then we cannot insist that the domains of partial sections are open subsets (as it is otherwise customary in the definition of $\mathrm{TC}(X)$ or cat $(X)$ and as was originally proposed by Dranishnikov), because the topological complexity of such a map would be infinite. On the other hand, we are mostly interested in the topological complexity of relatively tame maps, whose singular sets are usually closed, so that our definition based on filtrations of $X \times Y$ by open sets works well (see also Section 3.4 for some general finiteness estimates for $\mathrm{TC}(f))$. Let us mention that if $f$ is a fibration, then we will show in Corollary 4.4 that the two approaches are equivalent. Furthermore, if $f$ is a fibration between compact ANR's, then by 
Theorem 4.6 $\mathrm{TC}(f)$ can be defined using arbitrary subsets of $X \times Y$ as domains for local sections to $\pi_{f}$.

The following alternative description of $\mathrm{TC}(f)$ is often used in applications.

Proposition 2.3. Let $f: X \rightarrow Y$ be any map. Then $\mathrm{TC}(f)$ equals the minimal integer $n$ such that there exists an increasing sequence of closed subsets

$$
\emptyset=C_{0} \subseteq C_{1} \subseteq C_{2} \subseteq \cdots \subseteq C_{n}=X \times Y,
$$

where $C_{i}-C_{i-1}$ admits a partial section of $\pi_{f}$ for $i=1, \ldots, n$.

Furthermore, if $X \times Y$ is locally compact, then $\mathrm{TC}(f)$ equals the minimal integer $n$ such that there exists a partition of $X \times Y$ into disjoint locally compact subsets $G_{1}, G_{2}, \ldots G_{n}$ where $G_{i}$ admits a partial section of $\pi_{f}$ for $i=1, \ldots, n$.

Proof. The equivalence of the open and closed definitions follows immediately from De Morgan's Laws and the fact that the complement of an open set is a closed set.

As for the second claim, recall that since $X \times Y$ is locally compact, then a subset $G$ is locally compact if and only if $G=C_{1}-C_{2}$ for some closed sets $C_{1}, C_{2}$. Therefore, given an increasing sequence

$$
\emptyset=C_{0} \subseteq C_{1} \subseteq \cdots \subseteq C_{n}=X \times Y,
$$

where $C_{i}-C_{i-1}$ admits a partial section of $\pi_{f}$, then the sets $G_{i}=C_{i}-C_{i-1}$ are disjoint, locally compact, and each $G_{i}$ admits a partial section of $\pi_{f}$.

To prove the converse, take a disjoint partition $X \times Y=G_{1} \sqcup G_{2} \sqcup \cdots \sqcup G_{n}$, where $G_{i}$ are locally compact and admit a partial section to $\pi_{f}$ and each $G_{i}$ as a difference $G_{i}=A_{i}-B_{i}$ of two closed sets. We can then define the following increasing sequence of closed sets:

$$
C_{1}=\bigcup_{i=1}^{n} B_{i} \quad \text { and } \quad C_{i}:=C_{i-1} \cup A_{i-1} \text { for } i=2, \ldots, n .
$$

Note that $C_{1}$ can also be expressed as $C_{1}=\bigcup_{i=1}^{n}\left(\bigcup_{j=1}^{n} G_{i} \cap B_{j}\right)$. Since $\bigcup_{j=1}^{n} G_{i} \cap$ $B_{j} \subset G_{i} \subset A_{i}$, we see that the sets $\bigcup_{j=1}^{n}\left(G_{i} \cap B_{j}\right)$ are separated from one another and so $C_{1}$ admits a partial section of $\pi_{f}$.

Furthermore, since $C_{i}-C_{i-1} \subset A_{i-1}-B_{i-1}$, we conclude that $C_{i}-C_{i-1}$ admit a partial section to $\pi_{f}$ for $i=1, \ldots, n$

Remark 2.4. Srinivasan [14] has recently proved that for $X$ a compact metric ANR one can equivalently define cat $(X)$ by partitioning $X$ into arbitrary categorical subsets. The proof is based on extensions of maps from a subset of $X$ to a suitably constructed open neighbourhood (cf. [14, Corollary 2.8]). Her approach can be extended to the case of topological complexity of a space, but the above examples show that even for very simple maps the choice of the domains for partial sections can greatly affect the outcome. We will return to this question in Section 4.

\section{Estimates of $\mathrm{TC}(f)$ for arbitrary maps}

From this point on we will assume that all spaces under consideration are metric absolute neighbourhood retracts (metric ANR's). As explained before, this will allow 
a direct comparison between the $\mathrm{TC}(f)$ and the category or topological complexity of its domain and codomain. The following simple lemma will be particularly useful for the comparison of the topological complexity of related maps.

Lemma 3.1. Let $f: X \rightarrow Y$ and $f^{\prime}: X^{\prime} \rightarrow Y^{\prime}$ be any maps, and suppose there exists a map $h: Y \rightarrow Y^{\prime}$ with the following property: whenever $f^{\prime}$ admits a partial section over some $A \subseteq Y^{\prime}, f$ admits a partial section over $h^{-1}(A)$ as depicted in the following diagram:

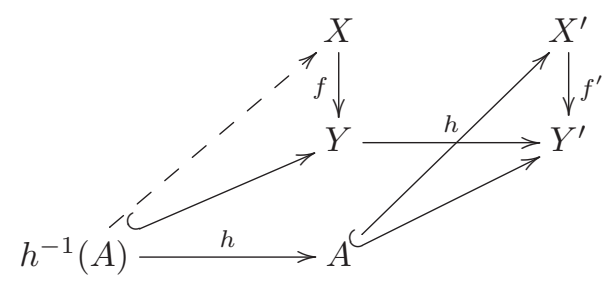

Then $\sec (f) \leqslant \sec \left(f^{\prime}\right)$.

Proof. Suppose that $\sec \left(f^{\prime}\right)=k$ and that

$$
U_{0} \subset U_{1} \subset \cdots \subset U_{k}=X^{\prime} \times Y^{\prime}
$$

is an increasing sequence of open subsets where $f^{\prime}$ admits a partial section over $U_{i}-$ $U_{i-1}$ for every $1 \leqslant i \leqslant k$. Then $f$ admits a partial section over each $h^{-1}\left(U_{i}-U_{i-1}\right)$ by hypothesis. Since $h$ is continuous, all $h^{-1}\left(U_{i}\right)$ are open and so

$$
\emptyset=h^{-1}\left(U_{0}\right) \subset h^{-1}\left(U_{1}\right) \subset \cdots \subset h^{-1}\left(U_{k}\right)=Y
$$

is an increasing sequence of open subsets where for every $1 \leqslant i \leqslant k$ the restriction of $f$ admits a continuous section over $h^{-1}\left(U_{i}\right)-h^{-1}\left(U_{i-1}\right)=h^{-1}\left(U_{i}-U_{i-1}\right)$. We conclude that $\sec (f) \leqslant k=\sec \left(f^{\prime}\right)$.

Proposition 3.2. For any map $f: X \rightarrow Y$, we have

$$
\mathrm{TC}(f) \geqslant \operatorname{cat}(Y) \text {. }
$$

Proof. Fix $x_{0} \in X$ and consider the inclusion $h: Y \hookrightarrow X \times Y$, given as $h(y):=\left(x_{0}, y\right)$. If $A \subseteq X \times Y$ admits a partial section $\sigma: A \rightarrow X^{I}$ to $\pi_{f}$, then one can easily check that

$$
\mathrm{ev}_{1} \circ f_{*} \circ \sigma \circ h=1_{h^{-1}(A)}
$$

where $f_{*}: P_{x_{0}} X \rightarrow P_{f\left(y_{0}\right)} Y$ denotes the post-composition by $f$. Therefore

$$
f_{*} \circ \sigma \circ h: h^{-1}(A) \rightarrow Y^{I}
$$

is a partial section to the map ev $1: P Y \rightarrow Y$ over $h^{-1}(A)$. By Lemma 3.1 we conclude that

$$
\mathrm{TC}(f)=\sec \left(\pi_{f}\right) \geqslant \sec \left(\mathrm{ev}_{1}\right)=\operatorname{cat}(Y) .
$$

As an easy consequence we obtain the relation

$$
\mathrm{TC}(f) \geqslant \operatorname{cat}(f),
$$

where cat $(f)$ denotes the Lusternik-Schnirelmann category of $f$ (see [1, Section 1.7] for 
the definition and main properties of cat $(f)$ ). This immediately follows from Proposition 3.2 and the fact that the category of a map is bounded above by the category of its codomain.

Another lower bound for $\mathrm{TC}(f)$ is given by the number of partial continuous sections of $f$.

Proposition 3.3. For any map $f: X \rightarrow Y$, we have

$$
\mathrm{TC}(f) \geqslant \sec (f) .
$$

In particular, if $\mathrm{TC}(f)=1$, then $f$ admits a continuous section.

Proof. Fix $x_{0} \in X$ and define $h: Y \rightarrow X \times Y$ as in the previous proof. If $\sigma: A \rightarrow Y^{I}$ is a partial section to $\pi_{f}$ then

$$
f \circ \mathrm{ev}_{1} \circ \sigma \circ h=1_{h^{-1}(A)},
$$

therefore $\mathrm{ev}_{1} \circ \sigma \circ h: h^{-1}(A) \rightarrow X$ is a partial section to $f$. By Lemma $3.1 \mathrm{TC}(f)=$ $\sec \left(\pi_{f}\right) \geqslant \sec (f)$.

Observe that if $f$ is a fibration, then $\sec (f) \leqslant \operatorname{cat}(Y)$, because $f$ admits a partial section over every categorical subset of $Y$. Therefore, for fibrations Proposition 3.2 implies Proposition 3.3.

Before proceeding let us introduce the following notation. Given a homotopy $H: X \times I \rightarrow Y$, we can use adjunction to define continuous functions $\vec{H}, \overleftarrow{H}: X \rightarrow Y^{I}$, by the formulas

$$
\vec{H}(x)(t):=H(x, t) \text { and } \overleftarrow{H}(x)(t):=H(x, 1-t)
$$

Proposition 3.4. If there exists $y_{0} \in Y$ such that the fibre $f^{-1}\left(y_{0}\right)$ of the map $f: X \rightarrow Y$ is categorical in $X$, then

$$
\mathrm{TC}(f) \geqslant \operatorname{cat}(X) \text {. }
$$

Proof. Define $h: X \rightarrow X \times Y$ by $h(x):=\left(x, y_{0}\right)$. By assumption, there exists a homotopy $H: f^{-1}\left(y_{0}\right) \times I \rightarrow X$ which deforms $f^{-1}\left(y_{0}\right)$ to a point. If $\sigma: A \rightarrow X^{I}$ is a partial section to $\pi_{f}$, then it is easy to verify that the map

$$
\vec{H} \circ \mathrm{ev}_{1} \circ \sigma \circ h
$$

determines a deformation of $h^{-1}(A) \subseteq X$ to a point in $X$. As before, by Lemma 3.1 we conclude that $\mathrm{TC}(f) \geqslant \operatorname{cat}(X)$.

\subsection{Effect of pre-composition on the complexity}

Our next objective is to study the effect that pre-composition by a map has on the complexity of $f$.

Theorem 3.5. Consider the diagram $\widehat{X} \stackrel{v}{\rightarrow} X \stackrel{f}{\rightarrow} Y$.

a) If $v$ admits a right homotopy inverse (i.e., a map $u: Y \rightarrow X$, such that $v u \simeq 1$ ), then $\mathrm{TC}(f v) \geqslant \mathrm{TC}(f)$

b) If $v$ admits a left homotopy inverse (a map $u$ such that $u v \simeq 1$ ) and if $f v u=f$, then $\mathrm{TC}(f v) \leqslant \mathrm{TC}(f)$. 
c) If $v$ admits a left homotopy inverse $u$, if $f v u \simeq f$ and if additionally $f v$ is a fibration, then $\mathrm{TC}(f v) \leqslant \mathrm{TC}(f)$

Proof. a) Suppose $A \subset \widehat{X} \times Y$ admits a partial section of $\pi_{f v}$, say $\alpha_{f v}: A \rightarrow \widehat{X}^{I}$ and $H: v u \simeq 1$. Then the formula

$$
\alpha_{f}(y, z):=\overleftarrow{H}(y) \cdot\left(v \circ \alpha_{f v}(u(y), z)\right)
$$

defines a continuous partial section on $(u \times 1)^{-1}(A)$. Since $(u \times 1): X \times Y \rightarrow$ $\widehat{X} \times Y$ is continuous, then $\mathrm{TC}(f) \leqslant \mathrm{TC}(f v)$ by Lemma 3.1 .

b) Suppose $A \subset X \times Y$ admits a partial section of $\pi_{f}$, say $\alpha_{f}: A \rightarrow X^{I}$. Let $H: u v \simeq 1$. Then the formula

$$
\alpha_{f v}(x, z):=\overleftarrow{H}(x) \cdot\left(u \circ \alpha_{f}(v(x), z)\right)
$$

defines a continuous map on $(v \times 1)^{-1}(A)$. Observe that $\alpha_{f v}(x, z)$ is path starting at $x$ and ending at $u\left(y^{\prime}\right)$ where $f\left(y^{\prime}\right)=z$. Thus $f v \circ \alpha_{f v}(x, z)$ ends at $f v\left(u\left(y^{\prime}\right)\right)=f\left(y^{\prime}\right)=z$. Therefore $\alpha_{f v}$ is a continuous partial section for $\pi_{f v}$. Again, $(v \times 1): \widehat{X} \times Y \rightarrow X \times Y$ is continuous, so, by 3.1, $\mathrm{TC}(f v) \leqslant \mathrm{TC}(f)$.

c) Suppose $A \subset X \times Y$ admits a partial section of $\pi_{f}$, say $\alpha_{f}: A \rightarrow X^{I}$. Let $H: u v \simeq 1$ and $K: f v u \simeq f$. Let $\Gamma_{f v}: \widehat{X} \sqcap Y^{I} \rightarrow X^{I}$ denote the lifting function for the fibration $f v$. Then the formula

$$
\alpha_{f v}(x, z):=\overleftarrow{H}(x) \cdot\left(u \circ \alpha_{f}(v(x), z)\right) \cdot \Gamma_{f v}\left(u\left(x^{\prime}\right), \vec{K}\left(x^{\prime}\right)\right)
$$

where $x^{\prime}=\alpha_{f}(v(x), z)(1)$, defines a continuous partial section for $(v \times 1)^{-1}(A)$. Thus by $3.1 \mathrm{TC}(f v) \leqslant \mathrm{TC}(f)$.

Furthermore, we have the following surprising result that the complexity of a map cannot increase if we pre-compose it with a fibration.

Theorem 3.6. If $v: X \rightarrow Y$ is a fibration, then $\mathrm{TC}(f v) \leqslant \mathrm{TC}(f)$ for every $f: X \rightarrow Y$.

Proof. Let $\alpha_{f}: A \rightarrow Y^{I}$ be a partial section for $\pi_{f}: P Y \rightarrow Y \times Z$ over some $A \subseteq$ $Y \times Z$. Then the formula

$$
\alpha_{f v}(x, y):=\Gamma_{v}\left(x, \alpha_{f}(v(x), z)\right)
$$

defines a partial section for $\pi_{f v}$ over $(v \times 1)^{-1}(A)$. As usual, this implies that $\mathrm{TC}(f v) \leqslant \mathrm{TC}(f)$,

The above theorems have several interesting corollaries. First, we deduce the following important invariance property, which states that the complexity of the map is not altered by a deformation retraction of the domain.

Corollary 3.7. If $v: \widehat{X} \rightarrow X$ is a deformation retraction, then for every $f: X \rightarrow Y$ we have $\mathrm{TC}(f)=\mathrm{TC}(f v)$.

Proof. Let $i: X \hookrightarrow \widehat{X}$ be the inclusion, so that $v i=1_{X}$ and $i v \simeq 1_{\widehat{X}}$. Then Theorem 3.5(a) implies that $\mathrm{TC}(f v) \geqslant \mathrm{TC}(f)$, while statement (b) and the observation that $f h i=f$ gives $\mathrm{TC}(f h) \leqslant \mathrm{TC}(f)$. 
It is important to keep in mind that the deformation retraction in the statement of the above Corollary cannot be replaced by an arbitrary homotopy equivalence. For example, the identity map $1_{[0,2]}$ and the map $f$ depicted in Figure 1 have homotopy equivalent domains, and yet the complexity of $f$ is $\operatorname{TC}(f)=2$, while $\operatorname{TC}\left(1_{[0,2]}\right)=1$. The problem is that a homotopy equivalence $u$ between the domains cannot be chosen so to be a fibrewise map over the base $[0,2]$, i.e. so that the following diagram strictly commutes:

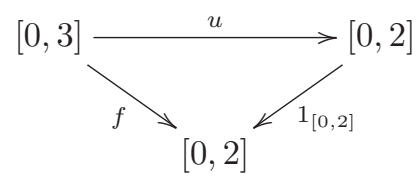

Nevertheless, if $f: X \rightarrow Y$ is a homotopy equivalence, then Corollary 3.8(b) bellow applies so we have $\mathrm{TC}(f) \geqslant \mathrm{TC}(X)=\mathrm{TC}(Y)$.

Corollary 3.8. a) If $f: X \rightarrow Y$ is a fibration, then $\mathrm{TC}(f) \leqslant \mathrm{TC}(Y)$.

b) If $f: X \rightarrow Y$ admits a homotopy section, then $\mathrm{TC}(f) \geqslant \mathrm{TC}(Y)$.

c) If $f: X \rightarrow Y$ is a fibration that admits a homotopy section, then $\mathrm{TC}(f)=$ $\mathrm{TC}(Y)$.

Proof. Consider the following diagram:

$$
X \stackrel{f}{\longrightarrow} Y \stackrel{1_{Y}}{=} Y .
$$

If $f$ is a fibration, then by Theorem 3.6

$$
\mathrm{TC}(f)=\mathrm{TC}\left(1_{Y} \circ f\right) \leqslant \mathrm{TC}\left(1_{Y}\right)=\mathrm{TC}(Y) .
$$

On the other hand, if $f$ admits a homotopy section $s: Y \rightarrow X$, then by Theorem 3.5(a)

$$
\mathrm{TC}(f)=\mathrm{TC}\left(1_{Y} \circ f\right) \geqslant \mathrm{TC}\left(1_{Y}\right)=\mathrm{TC}(Y) .
$$

By putting together (a) and (b) we get (c).

\subsection{Invariance with respect to homotopy}

Recall that two maps $f: X \rightarrow Y$ and $f^{\prime}: X^{\prime} \rightarrow Y$ are said to be fibre homotopy equivalent (or FHE-equivalent) if there is a commutative diagram of the form

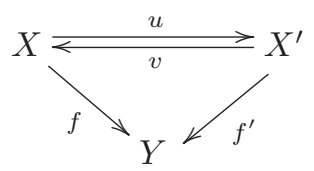

and the maps $u \circ v$ and $v \circ u$ are homotopic to the respective identity map by fibrepreserving homotopies. It is not surprising that topological complexities of fibrehomotopic maps are equal. In fact, a little more is true:

Corollary 3.9. Given $f: X \rightarrow Y$ and $g: X^{\prime} \rightarrow Y$ assume that there exist fibrewise maps $u: X \rightarrow X^{\prime}$ and $v: X^{\prime} \rightarrow X$ that homotopy inverses one to the other. Then $\mathrm{TC}(f)=\mathrm{TC}\left(f^{\prime}\right)$.

In particular, the topological complexity is a FHE-invariant. 
Proof. By Theorem 3.5(a) we have

$$
\mathrm{TC}(f)=\mathrm{TC}\left(f^{\prime} u\right) \geqslant \mathrm{TC}\left(f^{\prime}\right)=\mathrm{TC}(f v) \geqslant \mathrm{TC}(f),
$$

therefore $\operatorname{TC}(f)=\operatorname{TC}\left(f^{\prime}\right)$.

The following proposition shows that the fibrations have minimal complexity within their homotopy class.

Proposition 3.10. If $f \simeq g: X \rightarrow Y$ and $f$ is a fibration, then $\mathrm{TC}(f) \leqslant \mathrm{TC}(g)$.

Proof. Let $H: f \simeq g$, and let $\Gamma: X \sqcap Y^{I} \rightarrow X^{I}$ denote the lifting function for the fibration $f$.

Suppose $A \subset X \times Y$ admits a partial section of $\pi_{g}$, say $\alpha: A \rightarrow X^{I}$. Then for every $(x, y) \in A, \alpha(x, y)$ is a path in $X$ starting at $x$ and ending at $x^{\prime}$ such that $g\left(x^{\prime}\right)=y$. Observe that $x^{\prime}=\operatorname{ev}_{1}(\alpha(x, y))$ is continuously dependent on $(x, y)$.

Define $\bar{\alpha}(x, y):=\alpha(x, y) \cdot \Gamma\left(x^{\prime}, \vec{H}\left(x^{\prime}\right)\right)$. Clearly, $\bar{\alpha}$ is a continuous section of $(1 \times$ $f) \circ \mathrm{ev}_{0,1}$. Thus by $3.1, \mathrm{TC}(f) \leqslant \mathrm{TC}(g)$.

In particular, we have

Corollary 3.11. If $f, g: X \rightarrow Y$ are homotopic fibrations, then $\mathrm{TC}(f)=\mathrm{TC}(g)$.

Another important consequence of Theorem 3.5 is that the complexity cannot increase if we replace a map by a fibration.

Corollary 3.12. If $\bar{f}: \bar{X} \rightarrow Y$ is the fibrational substitute for $f: X \rightarrow Y$, then $\mathrm{TC}(\bar{f}) \leqslant \mathrm{TC}(f)$. Equality holds if $f$ is a fibration.

Proof. Since $\bar{f}$ is the fibrational substitute for $f$, we have the following diagram

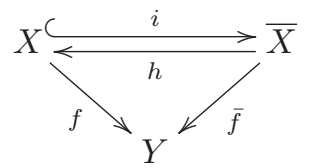

where $h$ is a fibration, $v u=1_{X}$ and $u v \simeq 1_{\bar{X}}$. Then the first claim follows by Theorem 3.5(a) because $\operatorname{TC}(\bar{f})=\mathrm{TC}(f h) \leqslant \mathrm{TC}(f)$. Moreover, if $f$ is a fibration, then so is $f h$, hence $\mathrm{TC}(f h) \geqslant \mathrm{TC}(f)$ Theorem $3.5(\mathrm{c})$.

\subsection{Effect of post-composition on the complexity}

Next we study the effect that the post-composition by a map has on the topological complexity.

Proposition 3.13. Consider the diagram $X \stackrel{f}{\rightarrow} Y \stackrel{v}{\rightarrow} \widehat{Y}$.

a) If $v$ admits a right inverse (section) $u: \widehat{Y} \rightarrow Y$, then $\operatorname{TC}(f) \geqslant \operatorname{TC}(v f)$

b) If $v$ admits a left homotopy inverse $u: \widehat{Y} \rightarrow Y$ and if $f$ is a fibration, then $\mathrm{TC}(f) \leqslant \mathrm{TC}(v f)$. 
Proof. a) Let $\pi_{f}: X^{I} \rightarrow X \times Y$ admit a partial section $\alpha: A \rightarrow X^{I}$ for some $A \subseteq$ $X \times Y$. Then the formula

$$
\alpha_{v f}(x, z):=\alpha_{f}(x,(u(z))
$$

defines a path starting at $x$ and ending at some $x^{\prime}$, such that $f\left(x^{\prime}\right)=u(z)$, therefore $v f\left(x^{\prime}\right)=v u(z)=z$. It follows that $\alpha_{v f}$ defines a partial section for $\pi_{v f}$ over $(1 \times u)^{-1}(A) \subseteq X \times \widehat{Y}$. As before, this implies $\mathrm{TC}(f) \geqslant \mathrm{TC}(v f)$.

b) Let $H: Y \times I \rightarrow Y$ be the homotopy from $u v$ to $1_{Y}$, and let $\alpha_{v f}: A \rightarrow X^{I} X$ be a partial section for $\pi_{v f}$ for some $A \subseteq X \times \widehat{Y}$. Then for every $(x, y) \in(1 \times v)^{-1}(A)$ the formula $\alpha_{f v}(x, v(y))$ gives a path in $X$ starting at $x$ and ending at some $x^{\prime}$, such that $v f\left(x^{\prime}\right)=v(y)$. Consequently $u v f\left(x^{\prime}\right)=u v(y)$, so $\overleftarrow{H}\left(f\left(x^{\prime}\right)\right) \cdot \vec{H}(y)$ is a path in $Y$ starting at $f\left(x^{\prime}\right)$ and ending at $y$. Therefore, the formula

$$
\alpha_{f}(x, y):=\alpha_{v f}(x, v(y)) \cdot \Gamma_{f}\left(x^{\prime}, \overleftarrow{H}\left(f\left(x^{\prime}\right)\right) \cdot \vec{H}(y)\right)
$$

defines a partial section to $\pi_{f}$ over $(1 \times v)^{-1}(A)$. Again, we conclude that $\mathrm{TC}(v f) \geqslant \mathrm{TC}(f)$.

The following result complements Corollary 3.8(b):

Corollary 3.14. If $f: X \rightarrow Y$ admits a section, then $\operatorname{TC}(f) \leqslant \mathrm{TC}(X)$.

Proof. Let $i: Y \rightarrow X$ be a right inverse for $f$ and apply Proposition 3.13 (a) to the diagram

$$
X \underset{=}{\stackrel{f}{\gtrless}} Y \text { i } Y
$$

Then we have

$$
\mathrm{TC}(f)=\mathrm{TC}\left(f \circ 1_{X}\right) \leqslant \mathrm{TC}\left(1_{X}\right)=\mathrm{TC}(X) .
$$

Observe, that the last result together with Corollary 3.8 yield the following very useful estimate: if $f: X \rightarrow Y$ admits a section, then

$$
\mathrm{TC}(X) \geqslant \mathrm{TC}(f) \geqslant \mathrm{TC}(Y) .
$$

The next result is analogous to Corollary 3.7, but it requires $f$ to be a fibration.

Corollary 3.15. If $v: Y \rightarrow \widehat{Y}$ is a deformation retraction then $\mathrm{TC}(v f)=\mathrm{TC}(f)$ for every fibration $f: X \rightarrow Y$.

Proof. By assumption, there is a map $i: \widehat{Y} \rightarrow Y$ such that $i v=1_{\widehat{Y}}$ and $v i \simeq 1_{Y}$. Then part (a) of Proposition 3.13 implies that $\mathrm{TC}(v f) \geqslant \mathrm{TC}(f)$, while part (b) implies $\mathrm{TC}(v f) \leqslant \mathrm{TC}(f)$.

In other words, if $f$ is a fibration, one cannot alter its complexity by deforming its codomain. This no longer needs to be true if $f$ is not a fibration. As an easy example, let $f:[0,3] \rightarrow[0,2]$ be the map consider before, and let $v:[0,2] \rightarrow[0,1]$ be given as

$$
v(t):=\left\{\begin{array}{cc}
t & t \in[0,1] \\
1 & t \in[1,2]
\end{array}\right.
$$

Clearly, $v$ is a deformation retraction and $\operatorname{TC}(v f)=1$, while $\operatorname{TC}(f)=2$. 
It is well-known (and easy to prove) that $\mathrm{TC}(X)=1$ if, and only if, $X$ is contractible. An analogous characterization of maps whose complexity is equal to 1 is more elusive.

Proposition 3.16. The following statements are equivalent for a map $f: X \rightarrow Y$ :

1. $\mathrm{TC}(f)=1$ and at least one fibre of $f$ is categorical in $X$.

2. $X$ is contractible and $f$ admits a continuous section.

Proof. Assume 1.: then by Proposition $3.3 f$ admits a continuous section, and by Proposition $3.4 \operatorname{cat}(X)=1$, therefore $X$ is contractible.

Conversely, if we assume 2., then Corollary 3.14 implies $\mathrm{TC}(f) \leqslant \mathrm{TC}(X)=1$, therefore $\mathrm{TC}(f)=1$.

However, note that if $Y$ is contractible then Corollary $3.8 \mathrm{~b}$ ) implies that the complexity of the projection pr: $Y \times F \rightarrow Y$ is equal to 1 regardless of the fibre $F$.

\subsection{A general upper bound for $\mathrm{TC}(f)$}

All upper estimates for $\mathrm{TC}(f)$ that we considered so far required quite restrictive assumptions on the map $f$ like being a fibration or admitting a (homotopy) section. The following theorem gives an upper estimate of $\mathrm{TC}(f)$ for general $f$.

Recall that subspaces $A, B$ of a topological space are said to be separated if $\bar{A} \cap B=$ $A \cap \bar{B}=\emptyset$. It is easy to verify that a function defined on $A \cup B$ is continuous if, and only if, its restrictions to $A$ and $B$ are continuous.

Theorem 3.17. Topological complexity of a map $f: X \rightarrow Y$ is bounded above by

$$
\mathrm{TC}(f) \leqslant \operatorname{cat}(X)+\sec (f)-1 .
$$

Proof. Let

$$
\emptyset=U_{0} \leqslant U_{1} \leqslant \ldots \leqslant U_{n}=X
$$

be an open filtration of $X$, such that for each $i$ the difference $U_{i}-U_{i-1}$ is categorical in $X$, i.e., there exists a homotopy $H_{i}: I \rightarrow X$ between the inclusion $U_{i} \hookrightarrow X$ and a constant map. Furthermore, let

$$
\emptyset=V_{0} \leqslant V_{1} \leqslant \ldots \leqslant V_{m}=Y
$$

be an open filtration of $Y$, such that on each difference $V_{j}-V_{j-1}$ there exists a continuous section $s_{j}: V_{j} \rightarrow X$ to $f$. The formula

$$
\sigma_{i, j}(x, y):=\vec{H}_{i}(x) \cdot \overleftarrow{H}_{i}\left(s_{j}(y)\right)
$$

clearly defines a partial section to $\pi_{f}$ over $U_{i} \times V_{j}$.

For every $2 \leqslant k \leqslant m+n$ let $W_{k}:=\bigcup_{i+j \leqslant k} U_{i} \times V_{j}$. Then

$$
W_{2} \subset W_{2} \subset \cdots W_{m+n}=X \times Y
$$

is an open filtration (of length $m+n-1$ ) of $X \times Y$ and for each $k$

$$
W_{k}-W_{k-1}=\bigcup_{i+j=k} U_{i} \times V_{j}
$$

Observe that the sets in the above union are separated, which implies that partial section $\sigma_{i, j}$ for $i+j=k$ define a continuous partial section on $W_{k}-W_{k-1}$. Since by 
definition of $\operatorname{cat}(X)$ and $\sec (f)$ we could assume $n=\operatorname{cat}(X)$ and $m=\sec (f)$ we have thus proved that $\mathrm{TC}(f) \leqslant \operatorname{cat}(X)+\sec (f)-1$.

The exact value of $\sec (f)$ is often hard to compute, so we mostly rely on the following coarser but easily computable estimate.

Corollary 3.18. Assume that the map $f: X \rightarrow Y$ is simplicial with respect to some choice of triangulations on $X$ and $Y$. Then

$$
\mathrm{TC}(f) \leqslant \operatorname{cat}(X)+\operatorname{dim}(Y)-1 .
$$

Proof. It is sufficient to prove that under the assumptions $\sec (f) \leqslant \operatorname{dim}(Y)$. To this end let $K$ and $L$ be simplicial complexes that triangulate respectively $X \approx|K|$ and $Y \approx|L|$, and with respect to which the map $f$ is simplicial. Consider the filtration of $Y$ by subcomplexes

$$
\left|L^{(0)}\right| \subset\left|L^{(1)}\right| \subset \cdots\left|L^{(\operatorname{dim} Y)}\right|=Y
$$

and observe that for every $1 \leqslant i \leqslant \operatorname{dim} Y$ the difference $\left|L^{(i)}\right|-\left|L^{(i-1)}\right|$ is a separated union of open $i$-simplices. Since the map $f$ is simplicial, it clearly admits a continuous section over each open $i$-simplex, and thus a continuous section over their separated union $\left|L^{(i)}\right|-\left|L^{(i-1)}\right|$. This shows that $\sec (f) \leqslant \operatorname{dim}(Y)$, which together with Theorem 3.17 implies our claim.

\subsection{Cohomological estimate of $\mathrm{TC}(f)$}

We mentioned in the Introduction the cohomological lower bound for the topological complexity of a space

$$
\operatorname{TC}(X) \geqslant \operatorname{nil}\left(\left(\operatorname{ker} \Delta^{*}: H^{*}(X \times X) \rightarrow H^{*}(X)\right),\right.
$$

which is widely used in the computations of topological complexity. Here Ker $\Delta^{*}$ is the ideal of 'zero divisors' (cf. [4]) and its nilpotency nil(Ker $\Delta^{*}$ ) is the minimal integer $n$ for which every product of $n$ elements in Ker $\Delta^{*}$ is equal to zero. We will present a similar estimate for the topological complexity of a map (a variant of which was already used in $[\mathbf{1 0}])$.

Let $\sigma: A \rightarrow X^{I}$ be a partial section to $\pi_{f}: X^{I} \rightarrow X \times Y$ and consider the following diagram:

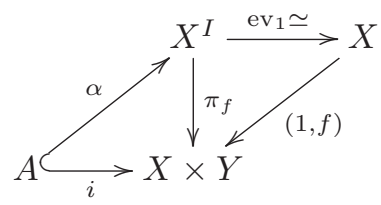

in which the right-hand triangle is homotopy commutative. By applying any multiplicative cohomology functor $H^{*}$ and identifying $H^{*}\left(X^{I}\right)$ with $H^{*}(X)$ we obtain a commutative diagram:

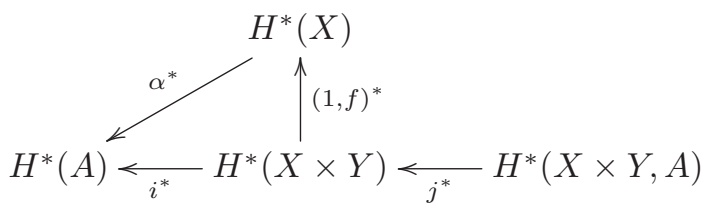


in which the bottom row is exact. It follows that every class $u \in \operatorname{Ker}(1, f)^{*}$ is contained in $\operatorname{Ker} i^{*}=\operatorname{Im} j^{*}$, so it is of the form $u=j^{*}(\bar{u})$ for some relative class $\bar{u} \in H^{*}(X \times$ $Y, A)$. If $A_{1}, \ldots, A_{n}$ is a cover of $X \times Y$ by sets that admit local sections to $\pi_{f}$, then there are relative classes $\bar{u}_{1} \in H^{*}\left(X \times Y, A_{1}\right) \ldots \bar{u}_{n} \in H^{*}\left(X \times Y, A_{n}\right)$ such that $u_{i}=j^{*}\left(\bar{u}_{i}\right)$. By the properties of the cohomology product we obtain

$$
u_{1} \cdot \ldots \cdot u_{n}=j^{*}\left(\bar{u}_{1} \cdot \ldots \cdot \bar{u}_{n}\right)=0
$$

because $\bar{u}_{1} \cdot \ldots \cdot \bar{u}_{n} \in H^{*}\left(X \times Y, A_{1} \cup \cdots \cup A_{n}\right)=H^{*}(X \times Y, X \times Y)=0$. We conclude that the product of any $n$ classes in $\operatorname{Ker}\left((1, f)^{*}\right)$ must be zero.

Theorem 3.19. For every map $f: X \rightarrow Y$ and for every multiplicative cohomology theory $H^{*}$ we have the estimate

$$
\mathrm{TC}(f) \geqslant \operatorname{nil}\left(\operatorname{Ker}(1, f)^{*}: H^{*}(X \times Y) \rightarrow H^{*}(X)\right) .
$$

Although the theorem is formulated in general terms, we will mostly consider the cases when $H^{*}(X \times Y) \cong H^{*}(X) \otimes H^{*}(Y)$. Then the action of $(1, f)^{*}$ on decomposable tensors is given as

$$
u \in X^{*}(X), v \in H^{*}(Y), \quad(1, f)^{*}(u \otimes v)=u \cdot f^{*}(v) \in H^{*}(X) .
$$

Normally we do not attempt to compute the entire kernel of the homomorphism $(1, f)^{*}$ but we rather look for specific elements in the kernel and try to find long non-trivial products. A common source of elements in $\operatorname{Ker}(1, f)^{*}$ are classes of the form $f^{*}(v) \otimes 1-1 \otimes v$ for $v \in H^{*}(Y)$.

\subsection{Summary of main estimates}

For the convenience of the reader, we summarize in one place the main estimates for the topological complexity of an arbitrary map.

Let $f: X \rightarrow Y$ be any map.

- $\max \{\operatorname{cat}(Y), \sec (f)\} \leqslant \mathrm{TC}(f) \leqslant \operatorname{cat}(X)+\sec (f)-1$

- $f$ simplicial $\Rightarrow \mathrm{TC}(f) \leqslant \operatorname{cat}(X)+\operatorname{dim}(Y)-1$

- $f$ admits a section $\Rightarrow \mathrm{TC}(Y) \leqslant \mathrm{TC}(f) \leqslant \mathrm{TC}(X)$

- $f$ fibration $\Rightarrow \mathrm{TC}(f) \leqslant \mathrm{TC}(Y)$

- $\mathrm{TC}(f)$ is FHE invariant

- $v: \widehat{X} \rightarrow X$ deformation retraction $\Rightarrow \mathrm{TC}(f)=\mathrm{TC}(f v)$

- $f \simeq g, g$ fibration $\Rightarrow \mathrm{TC}(g) \leqslant \mathrm{TC}(f)$

- $\bar{f}$ fibrational substitute for $f \Rightarrow \mathrm{TC}(\bar{f}) \leqslant \mathrm{TC}(f)$

- $\mathrm{TC}(f) \geqslant \operatorname{nil}\left(\operatorname{Ker}(1, f)^{*}: H^{*}(X \times Y) \rightarrow H^{*}(X)\right)$

For completeness we state without proof the following estimates (see [11, Proposition 5.5 and Theorem 6.1]).

- Product formula: for $f: X \rightarrow Y$ and $f^{\prime}: X^{\prime} \rightarrow Y^{\prime}$ we have

$$
\max \left\{\mathrm{TC}(f), \operatorname{TC}\left(f^{\prime}\right)\right\} \leqslant \mathrm{TC}\left(f \times f^{\prime}\right) \leqslant \mathrm{TC}(f)+\mathrm{TC}\left(f^{\prime}\right)-1 .
$$

- For every partition $X \times Y=G_{1} \sqcup \ldots \sqcup G_{n}$ into disjoint subsets admitting a partial section to $\pi_{f}$ there exists a point $(x, y) \in X \times Y$ such that every neighbourhood of it intersects at least $\mathrm{TC}(f)$ different domains $G_{i}$. 


\section{Topological complexity of a fibration}

As seen in the previous sections, several results about topological complexity depend on the assumption that some of the maps involved are fibrations. We will now explore this situation more thoroughly. Furthermore, as explained in Section 2, the invariants sec and $\mathrm{sec}_{\mathrm{op}}$ for fibrations whose base is an ANR. We will thus reiterate our standing assumption that $X$ and $Y$ are metric ANR's.

Lemma 4.1. The map $f: X \rightarrow Y$ is a fibration if, and only if, the induced map $\pi_{f}: X^{I} \rightarrow X \times Y$ is a fibration.

Proof. If $f$ is a fibration, then $1 \times f: X \times X \rightarrow X \times Y$ is also a fibration, thus $\pi_{f}$ can be written as a composition of two fibrations.

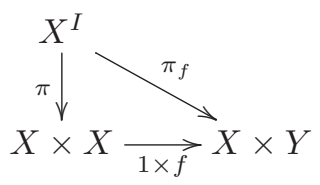

Conversely, assume $\pi_{f}$ is a fibration and consider arbitrary maps $h$ and $H$ for which the following diagram commutes

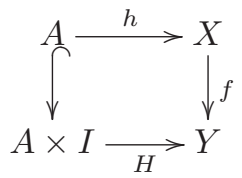

It gives rise to the following commutative diagram

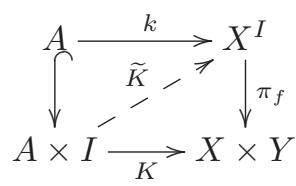

where $k(a)=$ const $_{a}, K(a, t)=(h(a), H(a, t))$, and $\widetilde{K}$ exists, because $\pi_{f}$ is a fibration. Then the map $\widetilde{H}: A \times I \rightarrow X$, defined by $\widetilde{H}(a, t):=\widetilde{K}(a, t)(1)$ is a suitable lifting of $H$ in the first diagram, which proves that $f$ is a fibration.

Since a homotopy section of a fibration can be always replaced by a strict section, we immediately obtain the following description of the topological complexity of a fibration.

Corollary 4.2. If $f: X \rightarrow Y$ is a fibration, then

$$
\operatorname{TC}(f):=\operatorname{secat}\left(\pi_{f}: X^{I} \rightarrow X \times Y\right) .
$$

It is often useful to restate the definition of $\mathrm{TC}(f)$ in more geometric terms, based on the following characterization (cf. [6, Lemma 4.2.1 and Proposition 4.2.4] for analogous description of $\mathrm{TC}(X))$.

Proposition 4.3. Let $f: X \rightarrow Y$ be a fibration, and let $A \subseteq X \times Y$. Then the following statements are equivalent: 
1. A admits a partial section $s: A \rightarrow X^{I}$ to the projection $\pi_{f}$;

2. The maps $f \circ \mathrm{pr}_{1}, \mathrm{pr}_{2}: A \rightarrow Y$ are homotopic;

3. A can be deformed in $X \times Y$ to the graph $\Gamma_{f}$ of the map $f$.

Proof. Let us denote by $\widehat{s}: A \times I \rightarrow X$ the adjoint of the partial section $s: A \rightarrow X^{I}$. Then $f \circ \widehat{s}: A \times I \rightarrow Y$ is clearly a homotopy between $f \circ \mathrm{pr}_{1}$ and $\mathrm{pr}_{2}$. Conversely, given a homotopy $H: A \times I \rightarrow Y$ between $f \circ \mathrm{pr}_{1}$ and $\mathrm{pr}_{2}$ one can use the fibration property to lift it to a homotopy $\widetilde{H}: A \times I \rightarrow X$, starting at $\widetilde{H}_{0}=\operatorname{pr}_{1}$. Then the adjoint of $\widetilde{H}$ is a partial section to $\pi_{f}$ over $A$.

In a similar vein, if $s: A \rightarrow X^{I}$ is a partial section to $\pi_{f}$, then we may define a homotopy $H: A \times I \rightarrow X \times Y$ as $H(a, t):=\left(s(a)\left(\frac{t}{2}\right), f\left(s(a)\left(1-\frac{t}{2}\right)\right)\right)$ and check that it defines a deformation of $A$ to $\Gamma_{f}$. On the other hand, let $H: A \times I \rightarrow X \times Y$ be a deformation of $A$ to $\Gamma_{f}$. Then we define a homotopy $K: A \times I \rightarrow Y$ by $K(a, t):=$ $\operatorname{pr}_{2}(H(a, 1-t))$ and lift it along the fibration $f$ to a homotopy $\widetilde{K}: A \times I \rightarrow X$ with $\widetilde{K}_{0}=\operatorname{pr}_{1}: A \rightarrow X$. It is easy to check that the adjoint of $\widetilde{K}$ is a partial section to $\pi_{f}$ over $A$.

Corollary 4.4. If $f: X \rightarrow Y$ is a fibration, then $\mathrm{TC}(f)$ equals the minimal number of elements of a covering of $X \times Y$ by open sets that can be deformed in $X \times Y$ to the graph of $f$.

As we mentioned in Remark 2.4, for a large class of spaces $X$ one can compute $\operatorname{cat}(X)$ and $\mathrm{TC}(X)$ by taking arbitrary subspaces of $X$ or $X \times X$ as domains of partial sections. We are going to show that an analogous result holds for the topological complexity of a fibration.

Lemma 4.5. Let $f, g: X \rightarrow Y$ be continuous maps between compact metric ANR spaces, and let $A$ be an arbitrary subset of $X$. If $\left.\left.f\right|_{A} \simeq g\right|_{A}$, then there exists an open neighbourhood $U \subseteq X$ of $A$ such that $\left.\left.f\right|_{U} \simeq g\right|_{U}$.

Proof. For simplicity we will use the same notation $d$ for the metrics in $X$ and $Y$ and also for the induced supremum metric on the space of path $Y^{I}$.

We will need the following standard properties of maps into metric ANR spaces:

- For every compact metric ANR space $E$ there exist an $\varepsilon>0$, such that every two maps $f, g: X \rightarrow E$ that are $\varepsilon$-close (i.e. $d(f(x), g(x))<\varepsilon$ for all $x \in X$ ) are homotopic (cf. [14, Theorem 2.4]).

- (Walsh lemma) Assume that $X$ and $E$ are separable metric spaces, and furthermore, that $E$ is an ANR. Let $h: A \rightarrow E$ be a continous map defined on an arbitrary subset $A \subseteq X$. Then, up to a small homotopy, $h$ can be extended to an open neighbourhood of $A$. More precisely, for every $\varepsilon, \delta>0$ there exists an open subset $U \subseteq X$ containing $A$ and a map $\bar{h}: U \rightarrow E$, satisfying the following conditions:

(1) for every $u \in U$ there exists $a \in A$ such that $d(u, a)<\delta$ and $d(\bar{h}(u), h(a))<\varepsilon$;

(2) $\left.\bar{h}\right|_{A} \simeq h$

(cf. [14, Theorem 2.3] and the comments at the end of the proof therein).

Returning to the proof of our statement, let $\varepsilon>0$ be such that any two $\varepsilon$-close maps $Y$ are homotopic. Since $X$ is compact, $f$ and $g$ are uniformly continuous, so there 
exists $\delta>0$ such that $d\left(x, x^{\prime}\right)<\delta$ imply $d\left(f(x), f\left(x^{\prime}\right)\right)<\frac{\varepsilon}{2}$ and $d\left(g(x), g\left(x^{\prime}\right)\right)<\frac{\varepsilon}{2}$. The homotopy $H: A \times I \rightarrow Y$ between $f$ and $g$ corresponds by adjunction to a map $\widehat{H}: A \rightarrow Y^{I}$. It is well-known that if $Y$ is a compact metric ANR then $Y^{I}$ is a metric ANR. Thus we may apply the Walsh lemma to obtain an open neighbourhood $U$ of $A$ and a map $G: U \rightarrow Y^{I}$, such that for every $u \in U$ there exists $a_{u} \in A$ satisfying $d\left(u, a_{u}\right)<\delta$ and $d\left(G(u), \widehat{H}\left(a_{u}\right)\right)<\frac{\varepsilon}{2}$ (i.e. $d\left(G(u)(t), \widehat{H}\left(a_{u}\right)(t)\right)<\frac{\varepsilon}{2}$ for all $\left.t \in I\right)$. Define $G_{0}, G_{1}: U \rightarrow Y$ as $G_{0}(u):=G(u)(0)$ and $G_{1}(u):=G(u)(1)$. Then for every $u \in U$ we have the triangle inequality (note that $H\left(a_{u}\right)(0)=f\left(a_{u}\right)$ )

$$
d\left(G_{0}(u), f(u)\right) \leqslant d\left(G_{0}(u), \widehat{H}\left(a_{u}\right)(0)\right)+d\left(f\left(a_{u}\right), f(u)\right)<\frac{\varepsilon}{2}+\frac{\varepsilon}{2}=\varepsilon .
$$

As a consequence, $G_{0}$ and $\left.f\right|_{U}$ are homotopic, and similarly for $G_{1}$ and $\left.g\right|_{U}$. Since $G_{0}$ and $G_{1}$ are homotopic by construction, we conclude that $\left.\left.f\right|_{U} \simeq g\right|_{U}$ as claimed.

Theorem 4.6. Let $f: X \rightarrow Y$ be a fibration between compact metric ANR spaces $X$ and $Y$. Then $\mathrm{TC}(f)$ is equal to the minimal integer $n$ for which there exists a cover

$$
X \times Y=A_{1} \cup \ldots \cup A_{n},
$$

such that each $A_{i}$ admits a continuous partial section to $\pi_{f}$.

Proof. It is clearly sufficient to show that each $A_{i}$ is contained in some open set that admits a partial section to $\pi_{f}$.

If $A_{i}$ admits a partial section to $\pi_{f}$ then the maps $f \circ \mathrm{pr}_{1}, \mathrm{pr}_{2}: A_{i} \rightarrow Y$ are homotopic by Proposition 4.3. Observe that $f \circ \mathrm{pr}_{1}$ and $\mathrm{pr}_{2}$ are defined on entire $X \times Y$. We may thus apply Lemma 4.5 to obtain an open neighbourhood $U_{i} \subseteq X \times Y$ of $A_{i}$, such that the maps $f \circ \mathrm{pr}_{1}, \mathrm{pr}_{2}: U_{i} \rightarrow Y$ are homotopic. Again by Proposition 4.3 it follows that $U_{i}$ admits a continuous partial section to $\pi_{f}$.

Most estimates of $\mathrm{TC}(f)$ can be considerably strengthened if we assume that $f$ is a fibration.

Proposition 4.7. If $f$ is a fibration then

$$
\operatorname{cat}(Y) \leqslant \mathrm{TC}(f) \leqslant \min \{\mathrm{TC}(Y), \operatorname{cat}(X \times Y)\} .
$$

In particular, $\mathrm{TC}(f)=1$ if, and only if $Y$ is contractible.

Proof. By Proposition 3.2 TC $(f) \geqslant \operatorname{cat}(Y)$, and by Corollary $3.8 \mathrm{TC}(f) \leqslant \mathrm{TC}(Y)$. Moreover, since $\pi_{f}$ is a fibration, there exists a partial section to $\pi_{f}$ over every categorical subset of $X \times Y$. As a consequence $\mathrm{TC}(f) \leqslant \operatorname{cat}(X \times Y)$.

If $Y$ is a topological group (or more generally, for an H-group), then the complexity of $Y$ coincide with its category, so we obtain the following result:

Corollary 4.8. Let $f: X \rightarrow Y$ be a fibration, and assume that $X$ is contractible or that $Y$ is an H-group. Then $\mathrm{TC}(f)=\operatorname{cat}(Y)$.

The following theorem allows a more detailed description of $\mathrm{TC}(f)$.

Theorem 4.9. a) If $f: X \rightarrow Y$ is a fibration, then the fibration $\pi_{f}: X^{I} \rightarrow X \times$ $Y$ is fibre-homotopy equivalent to the projection $q: X \sqcap Y^{I} \rightarrow X \times Y$ given by $q(x, \alpha):=(x, \alpha(1))$. 
b) Furthermore, the following diagram is a pull-back

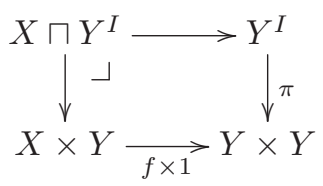

so in particular $q: X \sqcap Y^{I} \rightarrow X \times Y$ is a fibration with fibre $\Omega Y$.

As a consequence, if $f: X \rightarrow Y$ is a fibration, then $\mathrm{TC}(f)$ equals the sectional category of the fibration $q: X \sqcap Y^{I} \rightarrow X \times Y$.

Proof. a) Recall that $f: X \rightarrow Y$ is a fibration if, and only if, there exists a lifting function $\Gamma_{f}: X \sqcap P Y \rightarrow X^{I}$, which is, by definition, a section to the natural projection $p: X^{I} \rightarrow X \sqcap Y^{I}$, given by $p(\alpha)=(\alpha(1), f \circ \alpha)$. This may be restated by saying that $\Gamma_{f}$ and $p$ are fibrewise maps over $X \times Y$ as in the following commutative diagram (where $q(x, \alpha)=(x, \alpha(1))$ ).

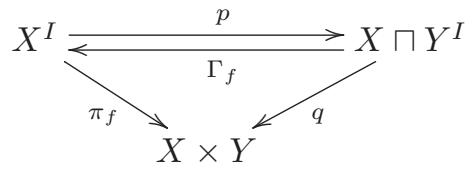

Since $p \circ \Gamma_{f}=1_{X \sqcap Y^{I}}$ and $\Gamma_{f} \circ p$ is fibre-homotopic to $1_{X^{I}}$ we conclude that $\pi_{f}$ and $p$ are fibre-homotopy equivalent.

b) The second statement follows from the following computation

$$
\begin{aligned}
(X \times Y) \sqcap Y^{I} & =\left\{(x, y, \alpha) \in X \times Y \times Y^{I} \mid f(x)=\alpha(0), y=\alpha(1)\right\} \\
& =\left\{(x, \alpha) \in X \times Y^{I} \mid f(x)=\alpha(0)\right\}=X \sqcap Y^{I}
\end{aligned}
$$

Being a pull-back of the path-fibration $\pi: Y^{I} \rightarrow Y \times Y$, the map $q$ is also a fibration, with the same fibre as $\pi$, which is the loop space $\Omega Y$.

We conclude the proof by observing that fibre-homotopy equivalent fibrations have the same sectional category.

It may be worth noting that we have actually proved that if $f: X \rightarrow Y$ is a fibration, then the diagram

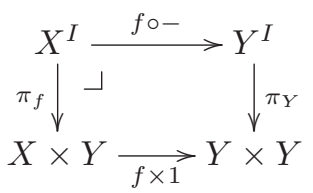

is a homotopy pull-back. Since the pull-back operation cannot increase sectional category, we immediately deduce $\mathrm{TC}(f)=\operatorname{secat}\left(\pi_{f}\right) \leqslant \operatorname{secat}\left(\pi_{Y}\right)=\mathrm{TC}(Y)$. On the other hand the sectional category of a fibration is smaller or equal to the category of the base, therefore $\mathrm{TC}(f) \leqslant \operatorname{cat}(X \times Y)$. We have thus obtained an alternative proof of Proposition 4.7.

Example 4.10. 1. $\mathrm{TC}(X \rightarrow\{y\})=1$, by Corollary 3.8(b).

2. $\mathrm{TC}\left(\mathrm{ev}_{1}: P X \rightarrow X\right)=\operatorname{cat}(X)$, by Corollary 4.8 . 
3. $\mathrm{TC}\left(\mathrm{ev}_{1}: X^{I} \rightarrow X\right)=\mathrm{TC}(X)$, by Corollary 3.8(b).

4. $\mathrm{TC}\left(\operatorname{pr}_{X}: X \times F \rightarrow X\right)=\mathrm{TC}(X)$ by Corollary 3.8(b). This example shows that the complexity of a map $f: X \rightarrow Y$ can be much smaller than cat $(X \times Y)$.

One very useful estimate of the topological complexity of a space is the "dimension divided by connectivity' bound (see [5]): if $X$ is $\operatorname{dim}(X)$-dimensional and $\operatorname{conn}(X)$ connected, then

$$
\mathrm{TC}(X) \leqslant\left\lfloor\frac{2 \operatorname{dim}(X)}{\operatorname{conn}(X)+1}\right\rfloor+1
$$

(where $\lfloor r\rfloor$ stands for the value of $r$ rounded down to the closest integer). The result is proved by obstruction theory applied to the Schwarz's [13] characterization of the sectional category. One could follow the same approach to estimate the sectional category of the fibration $q: X \sqcap P Y \rightarrow X \times Y$ with fibre $\Omega Y$, but it turns out that an even better estimate can be obtained by combining Proposition 4.7 with the dimension divided connectivity estimate for the category ([1, Theorem 1.50]).

Corollary 4.11. If $f: X \rightarrow Y$ is a fibration then

$$
\mathrm{TC}(f) \leqslant \min \left\{\left\lfloor\frac{\operatorname{dim}(X)}{\operatorname{conn}(X)+1}\right\rfloor,\left\lfloor\frac{\operatorname{dim}(Y)}{\operatorname{conn}(Y)+1}\right\rfloor\right\}+\left\lfloor\frac{\operatorname{dim}(Y)}{\operatorname{conn}(Y)+1}\right\rfloor+1,
$$

Proof. We may restate Proposition 4.7 as

$$
\mathrm{TC}(f) \leqslant \min \{\operatorname{cat}(X \times Y), \operatorname{cat}(Y \times Y)\} .
$$

Then the combination of the bound for the category of a product

$$
\operatorname{cat}(X \times Y) \leqslant \operatorname{cat}(X)+\operatorname{cat}(Y)-1,
$$

with the 'dimension divided connectivity' bound for the category $[\mathbf{1}]$

$$
\mathrm{TC}(f) \leqslant\left\lfloor\frac{\operatorname{dim}(X)}{\operatorname{conn}(X)+1}\right\rfloor
$$

yields the stated result.

Example 4.12. 1. Consider the covering map $p: S^{n} \rightarrow \mathbb{R} P^{n}$ : since dimension-toconnectivity ratio is smaller for the sphere than for the projective plane, Corollary 4.11 yields $\mathrm{TC}(p) \leqslant 1+n+1=n+2$. In comparison, $\mathrm{TC}\left(\mathbb{R} P^{n}\right)$ is usually much bigger and closer to $2 n$ (cf. [6]).

2. Similarly, for the standard quotient map $q: S^{2 n+1} \rightarrow \mathbb{C} P^{n}$ we obtain the estimate $\mathrm{TC}(q) \leqslant n+2$, which is much smaller that $\mathrm{TC}\left(\mathbb{C} P^{n}\right)=2 n+2$.

3. For a fibration over a sphere $f: X \rightarrow S^{n}$ we obtain $2=\operatorname{cat}\left(S^{n}\right) \leqslant \mathrm{TC}(f) \leqslant 3$. Observe that if $n$ is odd, we have $\mathrm{TC}(f)=2$ by Corollary 3.8, and the difference is caused by the fact that for odd-dimensional sphere the dimension-toconnectivity estimate is not sharp.

Let us illustrate the use of the cohomological estimate in the computation of the topological complexity of a map.

There are many fibrations for which $f^{*}: H^{*}(Y) \rightarrow H^{*}(X)$ is trivial (examples include $p: S^{n} \rightarrow \mathbb{R} P^{n}, q: S^{2 n+1} \rightarrow \mathbb{C} P^{n}$, Hopf fibrations, ...). In that case non-trivial 
elements in $\operatorname{Ker}(1, f)^{*}$ must be contained in $\oplus_{j>0} H^{i}(X) \otimes H^{j}(Y)$. It follows that every $k$-fold product in $\operatorname{Ker}(1, f)^{*}$ 'contains'a $k$-fold product in $H^{*}(Y)$, therefore

$$
\operatorname{nil}\left(\operatorname{Ker}(1, f)^{*}\right) \leqslant \operatorname{nil}\left(H^{*}(Y)\right) \leqslant \operatorname{cat}(Y),
$$

so if $f^{*}=0$ the cohomology estimate does not improve the estimate $\mathrm{TC}(f) \geqslant \operatorname{cat}(Y)$.

Example 4.13. Let $f: S O(n) \rightarrow S^{n-1}$ be the standard fibration obtained by projecting each orthogonal matrix to its last column. If $n$ is even, then

$$
2=\operatorname{cat}\left(S^{n-1}\right) \leqslant \mathrm{TC}(f) \leqslant \mathrm{TC}\left(S^{n-1}\right)=2,
$$

hence $\mathrm{TC}(f)=2$. However, if $n$ is odd, then $2 \leqslant \mathrm{TC}(f) \leqslant 3$, and we are going to use the cohomology estimate to show that the actual value is 3 . In fact, it is well known that the image $f^{*}(u)$ of a generator $u \in H^{n-1}\left(S^{n-1}\right)$ is a non-trivial element of $H^{n-1}(S O(n)$ because it reduces to one of the standard generators of $H *$ $n-1(S O(n) ; \mathbb{Z} / 2)$. Therefore $f^{*}(u) \otimes 1-1 \otimes u \in \operatorname{Ker}(1, f)^{*}$ and

$$
\left(f^{*}(u) \otimes 1-1 \otimes u\right)^{2}=-2 f^{*}(u) \otimes u \neq 0 .
$$

We conclude that $\mathrm{TC}(f)=3$.

The above example is an instance of a general situation when $f^{*}: H^{*}(Y) \rightarrow H^{*}(X)$ is injective. If we apply a cohomology functor $H^{*}$ to the following commutative diagram

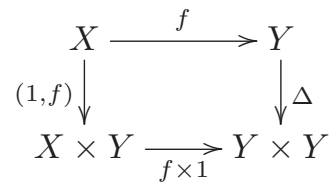

and assume that $H^{*}$ has field coefficients or that $H^{*}(Y)$ is free, and that $f^{*}$ is injective. Then we obtain the diagram

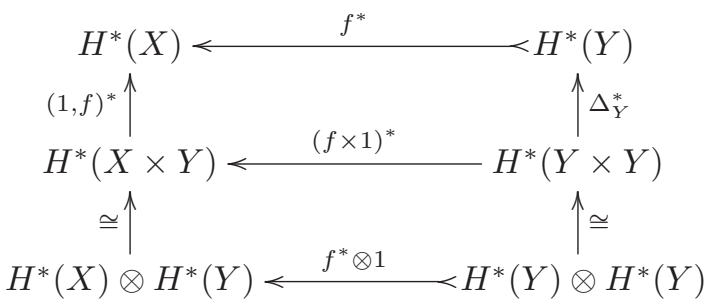

Observe that the $f^{*} \otimes 1$ is injective because we assumed that either $H^{*}$ has field coefficients or that $H^{*}(Y)$ is free, and tensoring with a free module preserves injectivity. The commutativity of the diagram implies that we can identify $\operatorname{Ker} \Delta_{Y}^{*}$ with a subideal of $\operatorname{Ker}(1, f)^{*}$, so we have proved the following result:

Theorem 4.14. Let $f: X \rightarrow Y$ be any map and assume that we consider a cohomology with field coefficients or that $H^{*}(Y)$ is free. If $f^{*}: H^{*}(Y) \rightarrow H^{*}(X)$ is injective, then $\mathrm{TC}(f) \geqslant \operatorname{nil}\left(\operatorname{Ker} \Delta_{Y}^{*}\right)$.

If, in addition, $f$ is a fibration, then $\operatorname{nil}\left(\operatorname{Ker} \Delta_{Y}^{*}\right) \leqslant \mathrm{TC}(f) \leqslant \mathrm{TC}(Y)$.

Note that the nilpotency of Ker $\Delta_{Y}^{*}$ was introduced by Farber [4] (under the name of 'zero divisors cup length') as the basic lower bound for the topological complexity. 
In many cases (in fact, in almost all cases where the exact value of $\mathrm{TC}(Y)$ is known) $\operatorname{nil}\left(\operatorname{Ker} \Delta_{Y}^{*}\right)$ is either equal to $\mathrm{TC}(Y)$ or to $\mathrm{TC}(Y)-1$, so the above estimate is a very useful tool for computations.

An important class of maps to which the above Theorem applies are fibre bundles whose fibres are totally non-homologous to zero. Recall that the fibre $F$ of a fibration $f: X \rightarrow Y$ is said to be totally non-homologous to zero with respect to a field $R$ if the homomorphism $H^{*}(X ; R) \rightarrow H^{*}(F ; R)$ induced by the inclusion of the fibre is surjective. If that case the Serre spectral sequence for $f$ collapses at the $E_{2}$-term, which in turn implies that $f^{*}: H^{*}(Y ; R) \rightarrow H^{*}(X ; R)$ is injective.

Corollary 4.15. If $f: X \rightarrow Y$ is a fibration whose fibre is totally non-homologous to zero with respect to a field $R$, and if $\mathrm{TC}(Y)=\operatorname{nil}\left(\operatorname{Ker} \Delta_{Y}^{*}\right)$ (cohomology with coefficients in $R)$, then $\mathrm{TC}(f)=\mathrm{TC}(Y)$.

Let $X$ be a pointed CW-complex (we omit the base-point from the notation), and let $\operatorname{Cov}(X)$ denote the set of (equivalence classes) of base-point preserving covering projections over $X$. It is well-known that there is a bijection between $\operatorname{Cov}(X)$ and the lattice of subgroups of the fundamental group $\pi_{1}(X)$. To every $G \leqslant \pi_{1}(X)$ there corresponds a unique $p_{G}: \widetilde{X}_{G} \rightarrow X$ such that $\operatorname{Im}\left(p_{G}\right)_{\sharp}=G$. In particular, $p_{\pi_{1}(X)}=$ $\operatorname{id}_{X}$ and $p_{\{1\}}$ is the universal covering projection over $X$.

If $G, G^{\prime}$ are subgroups of $\pi_{1}(X)$, then the lifting criterion for covering spaces implies that $G \leqslant G^{\prime}$ if, and only if, there exists a map $v: \widetilde{X}_{G} \rightarrow \widetilde{X}_{G^{\prime}}$ such that the following diagram commutes

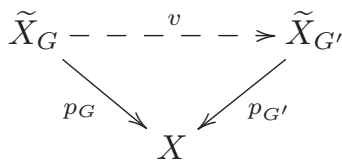

Moreover, when such $v$ exists it is unique and it is itself a covering projection. Therefore, if $G \leqslant G^{\prime} \leqslant \pi_{1}(X)$, then there is a fibration $v$ such that $p_{G^{\prime}} v=p_{G}$, and Theorem 3.6 implies that $\mathrm{TC}\left(p_{G}\right) \leqslant \mathrm{TC}\left(p_{G^{\prime}}\right)$. We have thus proved

Theorem 4.16. The topological complexity of covering projections determines an increasing map from the lattice of subgroups of $\pi_{1}(X)$ to $\mathbb{N} \cup\{\infty\}$. Its minimal value is the topological complexity of the universal covering projection and its maximal value is $\mathrm{TC}(X)$.

Observe that for an arbitrary covering projection $p: \widetilde{X} \rightarrow X$ Propositon 4.7 implies the estimate $\operatorname{cat}(X) \leqslant \mathrm{TC}(p) \leqslant \operatorname{cat}(X \times \widetilde{X})$, which is often easier to compute.

Let us now study more closely covering projections over Eilenberg-MacLane spaces. The homotopy type of an Eilenberg-MacLane space $K(G, 1)$ is uniquely determined by the group $G$. As a consequence both cat $(K(G, 1))$ and $\operatorname{TC}(K(G, 1))$ are in fact invariants of $G$ and are often denoted as cat $(G)$ and $\mathrm{TC}(G)$, respectively. Every covering projections over $K(G, 1)$ corresponds to a subgroup $H \leqslant G$ and its total space is in fact an Eilenberg-MacLane space of type $K(H, 1)$. Since the universal covering space of $K(G, 1)$ is contractible we have $\mathrm{TC}\left(p_{\{1\}}\right)=\operatorname{cat}(G)$ by 4.8 . Theorem 4.16 
then yields a general estimate

$$
\operatorname{cat}(G) \leqslant \mathrm{TC}(p: K(H, 1) \rightarrow K(G, 1)) \leqslant \mathrm{TC}(G) .
$$

Note that if $G$ is abelian then $K(G, 1)$ is an $H$-group and Corollary 4.8 implies that $\mathrm{TC}(p)=\operatorname{cat}(G)$ for every covering projection $p$ with base $K(G, 1)$.

We also give two non-commutative examples. Let $p: \widetilde{X} \rightarrow S^{1} \vee S^{1}$ be the universal covering of the wedge of two circles. Since $\widetilde{X}$ is contractible, we get $\operatorname{TC}(p)=2$, while $\mathrm{TC}\left(S^{1} \vee S^{1}\right)=3$. Similarly, let $S$ be a closed surface different from the sphere or projective plane, and let $p: \widetilde{S} \rightarrow S$ be its universal covering. Then $\widetilde{S}$ is contractible, therefore $\mathrm{TC}(p)=\operatorname{cat}(S)=3$ while $\mathrm{TC}(S)=5$.

Remark 4.17. Eilenberg and Ganea $[3]$ showed that cat $(G)$ can be expressed in a completely algebraic manner: they proved that $\operatorname{cat}(G)=\operatorname{cat}(K(G, 1)=\operatorname{cd}(G)+1$, where cd denotes the cohomological dimension of $G$.

At this moment there is no completely algebraic way to compute $\mathrm{TC}(G)$. We have the general estimate

$$
\operatorname{cd}(G)+1=\operatorname{cat}(K(G, 1)) \leqslant \mathrm{TC}(G) \leqslant \operatorname{cat}(K(G, 1) \times K(G, 1))=\operatorname{cd}(G \times G)+1 .
$$

Rudyak [12] proved that for a suitable choice of group $G$ the value of $\operatorname{TC}(G)$ can be any number between $\operatorname{cd}(G)+1$ and $\operatorname{cd}(G \times G)+1$. On the other hand it has been recently proved by Farber and Mescher [7] that for a large class of groups (including all hyperbolic groups) $\mathrm{TC}(G)$ is either $\operatorname{cd}(G \times G)$ or $\operatorname{cd}(G \times G)+1$.

We conclude with a partial result about finite-sheeted covering projections.

Theorem 4.18. Assume that the topological complexity of $X$ equals the rational cohomological lower bound $\mathrm{TC}(X)=\operatorname{nil}\left(\operatorname{ker} H^{*}(\Delta ; \mathbb{Q})\right)$. Then $\operatorname{TC}(p)=\operatorname{TC}(X)$ for every finite-sheeted covering projection $p: \widetilde{X} \rightarrow X$.

Proof. Recall that finite-sheeted covering projections induce monomorphisms in rational cohomology (see [8, Proposition 3G.1]). Then the claim follows directly from Theorem 4.14.

For instance, the topological complexity of every finite-sheeted covering over an orientable surface $P$ of genus bigger then 1 is equal to $\mathrm{TC}(P)=5$, while the topological complexity of its universal cover is equal to cat $(P)=3$. We do not know whether there are covering projections to $P$ whose topological complexity is 4 . On the other hand we suspect that $\mathrm{TC}(p)=\mathrm{TC}(X)$ for every finite sheeted covering projection $p$ with base $X$.

\section{Acknowledgments}

We are grateful to Nick Callor for helpful discussions on certain aspects of the article, in particular for his suggestion to base the definition of topological complexity on open filtrations. 


\section{References}

[1] O. Cornea, G. Lupton, J. Oprea, D. Tanré, Lusternik-Schnirelmann Category, AMS, Math. Surv. Monogr., vol. 103 (2003).

[2] A. Dranishnikov, On topological complexity of twisted products, Topology Appl. 179 (2015), 74-80.

[3] S. Eilenberg, T. Ganea,On the Lusternik-Schnirelmann Category of Abstract Groups, Ann. of Math. (2) 65 (1957), 517-518.

[4] M. Farber, Topological Complexity of Motion Planning, Discrete Comput. Geom., 29 (2003), 211-221.

[5] M. Farber, Instabilities of robot motion, Top. Appl. 140 (2004), 245-266

[6] M. Farber, Invitation to topological robotics, EMS Publishing House, Zurich, 2008.

[7] M. Farber, S. Mescher, On the topological complexity of aspherical spaces, to appear in J. Topol. Anal. https://doi.org/10.1142/S1793525319500511

[8] A. Hatcher, Algebraic Topology, Cambridge Univ. Press, Cambridge, 2002.

[9] P. Pavešić, Formal aspects of topological complexity, in A.K.M. Libardi (ed.), Zbirnik prac Institutu matematiki NAN Ukraini, ISSN 1815-2910, T. 6, (2013), $56-66$.

[10] P. Pavešić, Complexity of the forward kinematic map, Mech. Mach. Theory 117 (2017), 230-243.

[11] P. Pavešić, A topologist's view of kinematic maps and manipulation complexity, Contemp. Math. 702 (2018), 61-83.

[12] Y. Rudyak, On topological complexity of Eilenberg-MacLane spaces, Topology Proc. 48 (2016), 65-67.

[13] A.S. Schwarz, The genus of a fiber space, Amer. Math. Soc. Transl. Ser. (2) 55 (1966), 49-140.

[14] T. Srinivasan, On the Lusternik-Schnirelmann category of Peano continua, Topology Appl. 160 (2013), 1742-1749.

Petar Pavešić petar.pavesic@fmf.uni-lj.si

Faculty of Mathematics and Physics, University of Ljubljana, Jadranska 21, Ljubljana, Slovenia 